Supporting Information

\title{
Heterochiral Doped Supramolecular Coordination Networks for High-Performance Optoelectronics
}

Xiaobo Shang, ${ }^{\dagger, \perp}$ Inho Song,,$^{\dagger, \perp}$ Jeong Hyeon Lee, ${ }^{\ddagger}$ Wanuk Choi, ${ }^{\S}$ Hiroyoshi Ohtsu, ${ }^{\#}$ Gwan Yeong Jung, ${ }^{\ddagger}$ Jaeyong Ahn, ${ }^{\dagger}$ Myeonggeun Han, ${ }^{\|}$Jin Young Koo, ${ }^{\Delta}$ Masaki Kawano, ${ }^{\#}$ Sang Kyu Kwak, ${ }^{*}$, and Joon Hak Oh*,†

${ }^{\dagger}$ School of Chemical and Biological Engineering, Institute of Chemical Processes, Seoul National University, 1 Gwanak-ro, Gwanak-gu, Seoul 08826, Republic of Korea

tDepartment of Energy Engineering, School of Energy and Chemical Engineering, Ulsan National Institute of Science and Technology (UNIST), Ulsan 44919, Republic of Korea

${ }^{\S}$ Center for Ordered Nanoporous Materials Synthesis, Division of Environmental Science and Engineering, Pohang University of Science and Technology (POSTECH), Pohang, Gyeongbuk 37673, Republic of Korea

\#Department of Chemistry, School of Science, Tokyo Institute of Technology, 2-12-1 Ookayama, Meguro-ku, Tokyo 152-8550, Japan

'Department of Chemical Engineering, Pohang University of Science and Technology (POSTECH), Pohang, Gyeongbuk 37673, Republic of Korea

${ }^{\Delta}$ Department of Chemistry, Pohang University of Science and Technology (POSTECH), Pohang, Gyeongbuk 37673, Republic of Korea

*E-mail: skkwak@unist.ac.kr; joonhoh@snu.ac.kr 


\section{Supporting Contents}

Estimation of Optoelectrical Properties S-3

\section{Supporting Figures}

SEM images of AlaNDI-Cd SCNs (Figure S1) ------------------------------------------------S-4

Optical properties of ligand and AlaNDI-Cd SCNs (Figure S2) ---------------------------------S-5

TGA results of AlaNDI-Cd SCNs (Figure S3) ---------------------------------------------------S-6

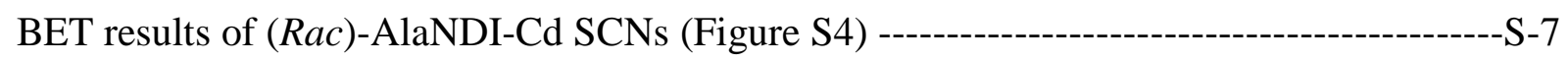

FT-IR and PXRD results before and after UV light irradiation (Figure S5) --------------------S-8

I-V characteristics of AlaNDI-Cd crystals under UV light exposure (Figure S6) --------S-9

FT-IR and PXRD results before and after hydrazine doping (Figure S7) --------------------S-10

I-V characteristics of hydrazine doped heterochiral AlaNDI-Cd crystals (Figure S8) ------ S-11

Time-dependent experiments for doped heterochiral AlaNDI-Cd crystals (Figure S9) ----- S-12

Cyclic stability of conductivity reversibility for doped AlaNDI-Cd crystals (Figure S10) - S-13

Temperature-dependent conductivity of heterochiral AlaNDI-Cd crystals (Figure S11) --- S-14

ESR spectra of $(S)$-AlaNDI-Cd crystals before and after hydrazine doping (Figure S12) -- S-15

Binding energy calculation for adsorbed hydrazine (Figure S13) ------------------------------S-16

Binding configurations of AlaNDI-Cd with adsorbed hydrazine (Figure S14) -------------- S-17 


\section{Estimation of Optoelectrical Properties}

In order to investigate optoelectronic properties for devices, photoresponsivity $(R)$ and photosensitivity $(P)$ were calculated from $I-V$ characteristics coupled with light irradiation. The $R$ and $P$ values are typically defined by the following equations:

$R=\frac{I_{\mathrm{ph}}}{P_{\text {inc }}}=\frac{I_{\text {light }}-I_{\text {dark }}}{P_{\text {inc }}}$

$P=\frac{I_{\text {light }}-I_{\text {dark }}}{I_{\text {dark }}}$

where $I_{\mathrm{ph}}$ is the photocurrent, $P_{\text {inc }}$ the incident illumination power on the channel of the device, $I_{\text {light }}$ the current under illumination, and $I_{\text {dark }}$ the current in the dark, respectively. In addition, the external quantum efficiency $(\mathrm{EQE})(\eta)$ of electronic devices was calculated which can be defined as the ratio of number of photogenerated carriers that practically enhances the current to the number of photons incident onto the device channel area, using the following equation (3):

$\eta=\frac{\left(I_{\text {light }}-I_{\text {dark }}\right) h c}{e P_{\text {int }} A \lambda_{\text {peak }}}$

where $h$ is the plank constant, $c$ the speed of light, $e$ the fundamental unit of charge, $A$ the area of the device channel, and $\lambda_{\text {peak }}$ the peak wavelength of the incident light, respectively. 

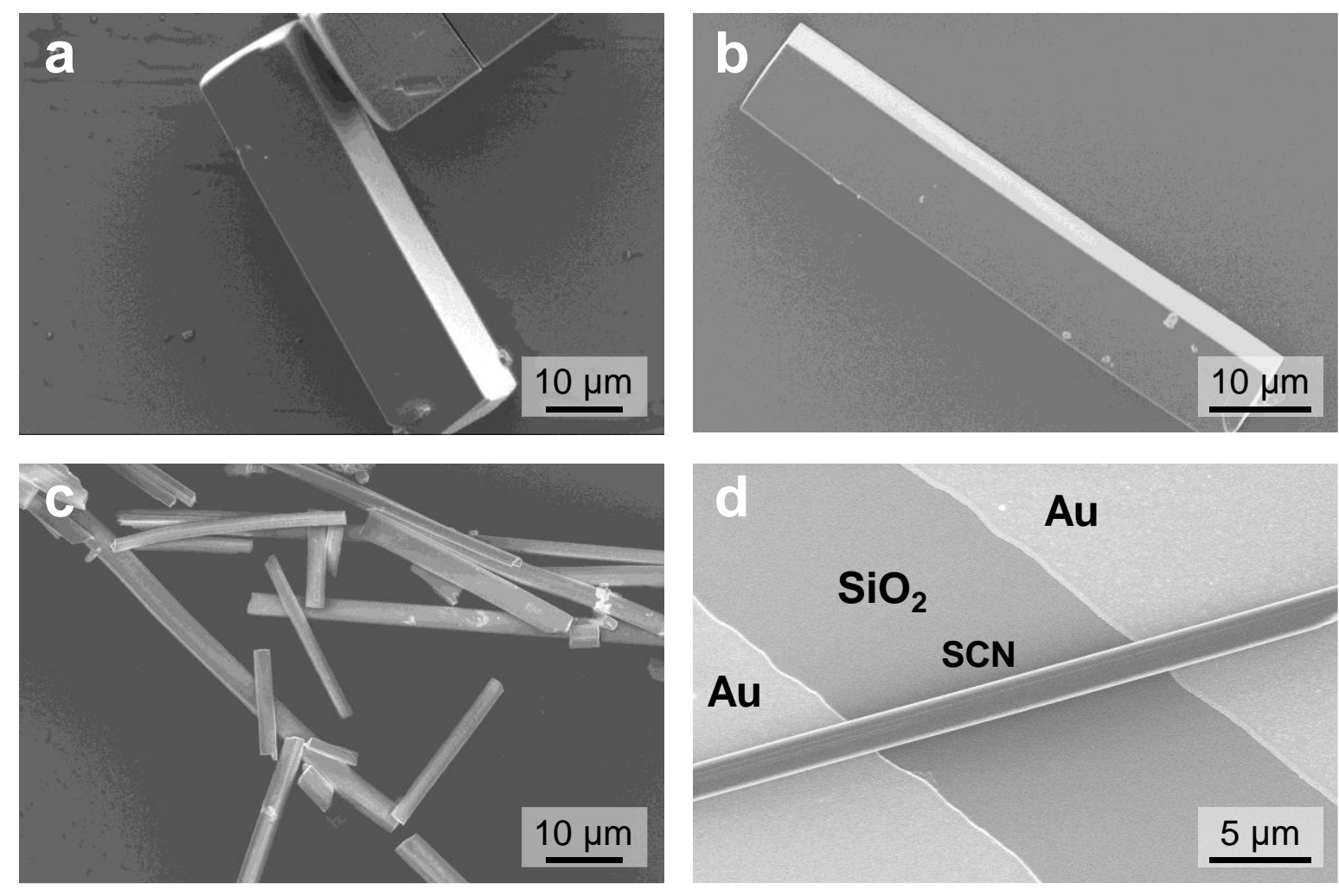

Figure S1. (a-d) SEM images of (a) (R)-AlaNDI-Cd, (b) (S)-AlaNDI-Cd, (c) (Rac)-AlaNDI$\mathrm{Cd}$, and (d) (Rac)-AlaNDI-Cd micro/nanocrystals on pre-patterned gold electrodes in electronic devices. 

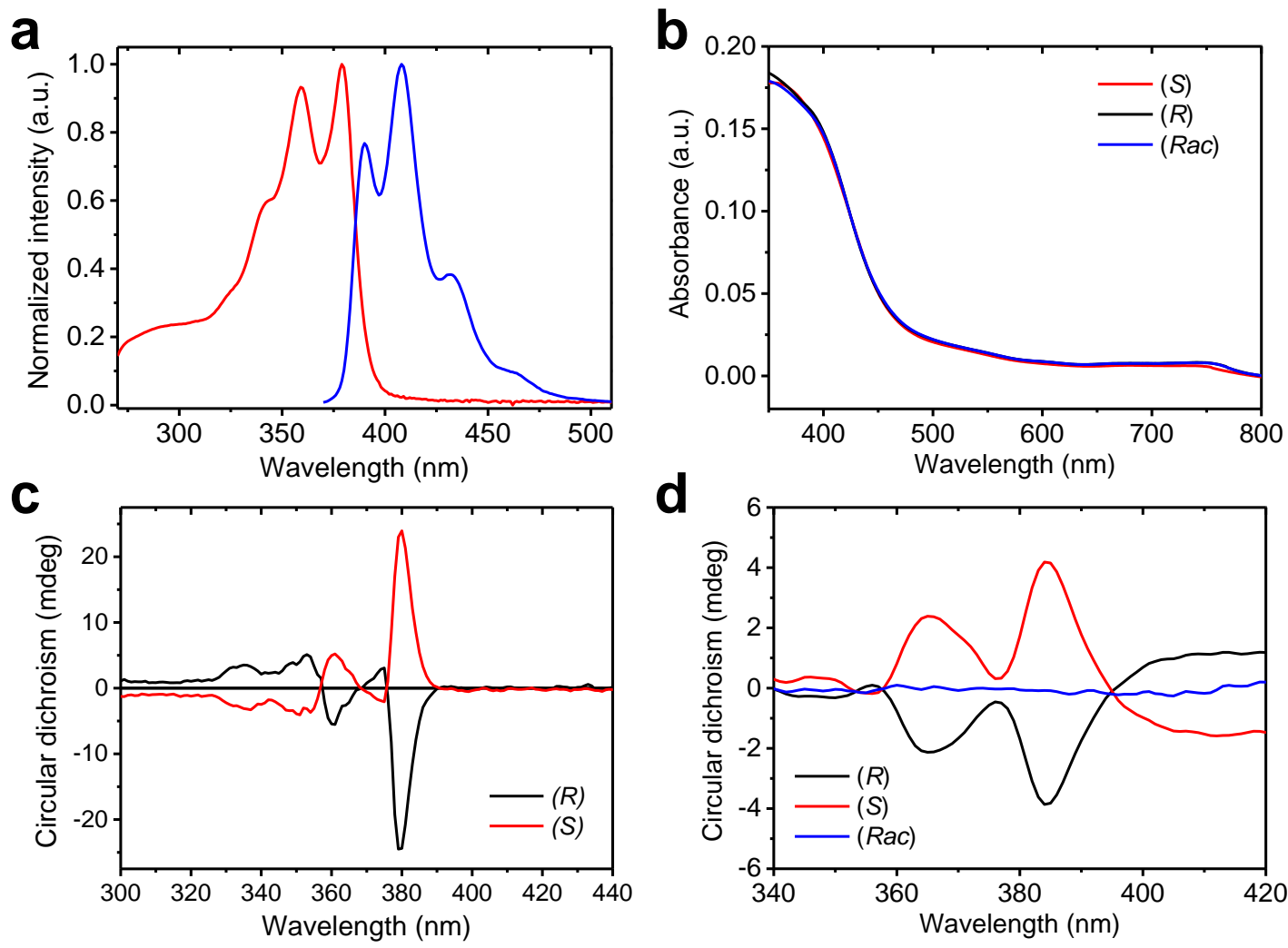

Figure S2. (a) UV-vis and PL spectra of $(S)-\mathrm{H}_{2}$ AlaNDI ligands. (b) UV-vis spectra of homochiral and heterochiral AlaNDI-Cd SCNs. (c) CD spectra of $(S)$ - and $(R)-\mathrm{H}_{2} \mathrm{AlaNDI}$ ligands. (d) CD spectra of homochiral and heterochiral AlaNDI-Cd SCNs. 


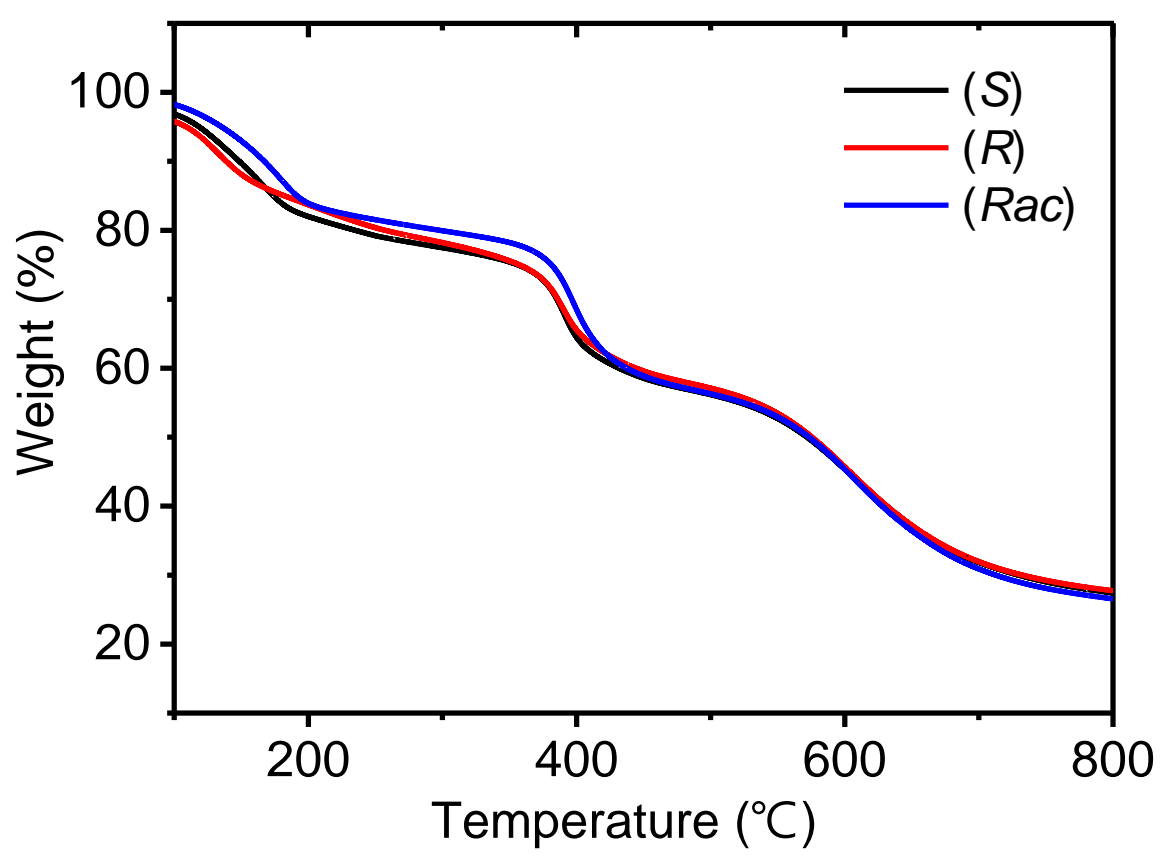

Figure S3. TGA results of $(R)$-AlaNDI-Cd, $(S)$-AlaNDI-Cd, and (Rac)-AlaNDI-Cd SCNs. 

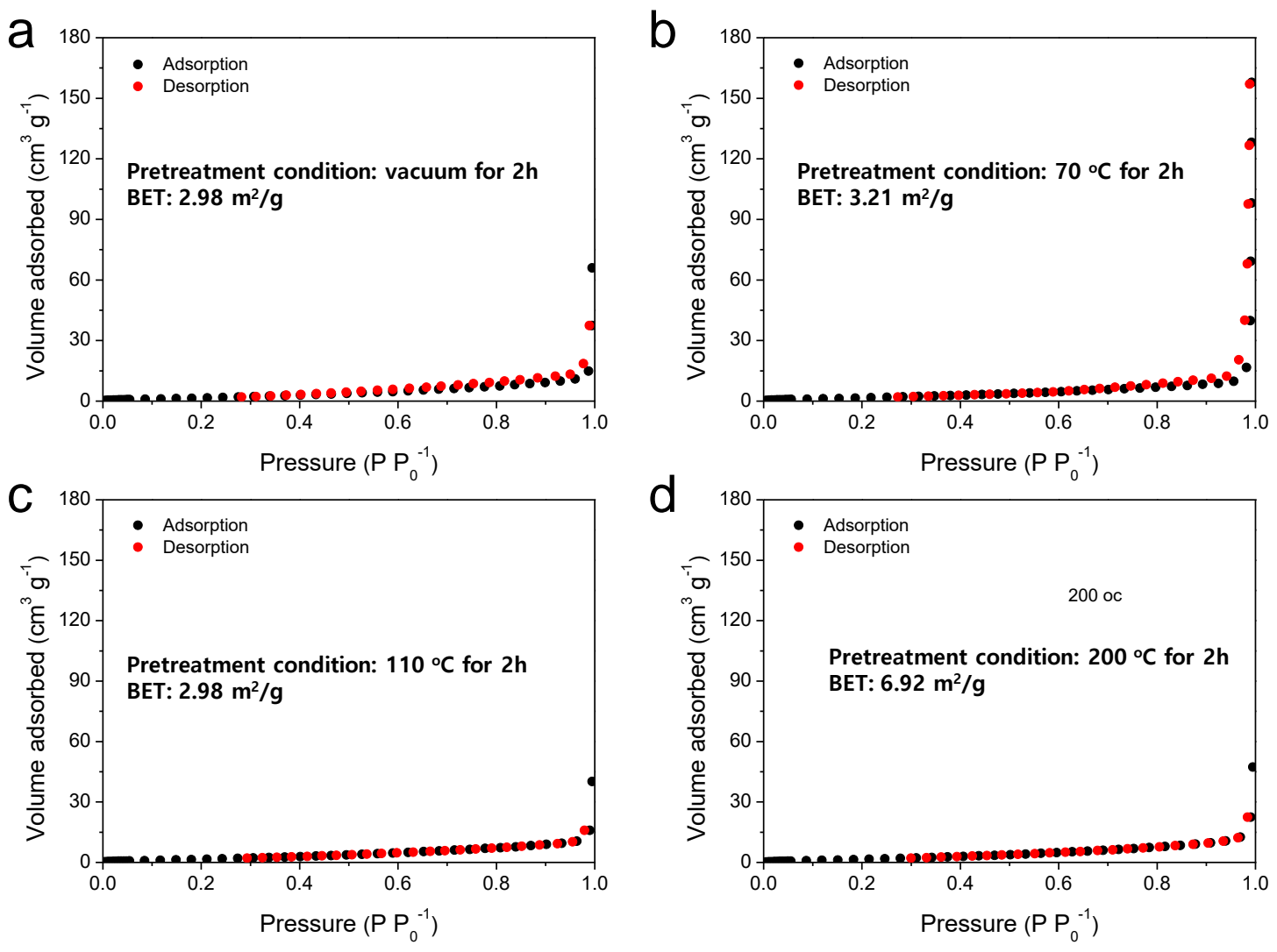

Figure S4. BET results of heterochiral (Rac)-AlaNDI-Cd SCNs (a) without heating, and after heating at (b) $70{ }^{\circ} \mathrm{C}$, (c) $110{ }^{\circ} \mathrm{C}$, and (d) $200{ }^{\circ} \mathrm{C}$. 

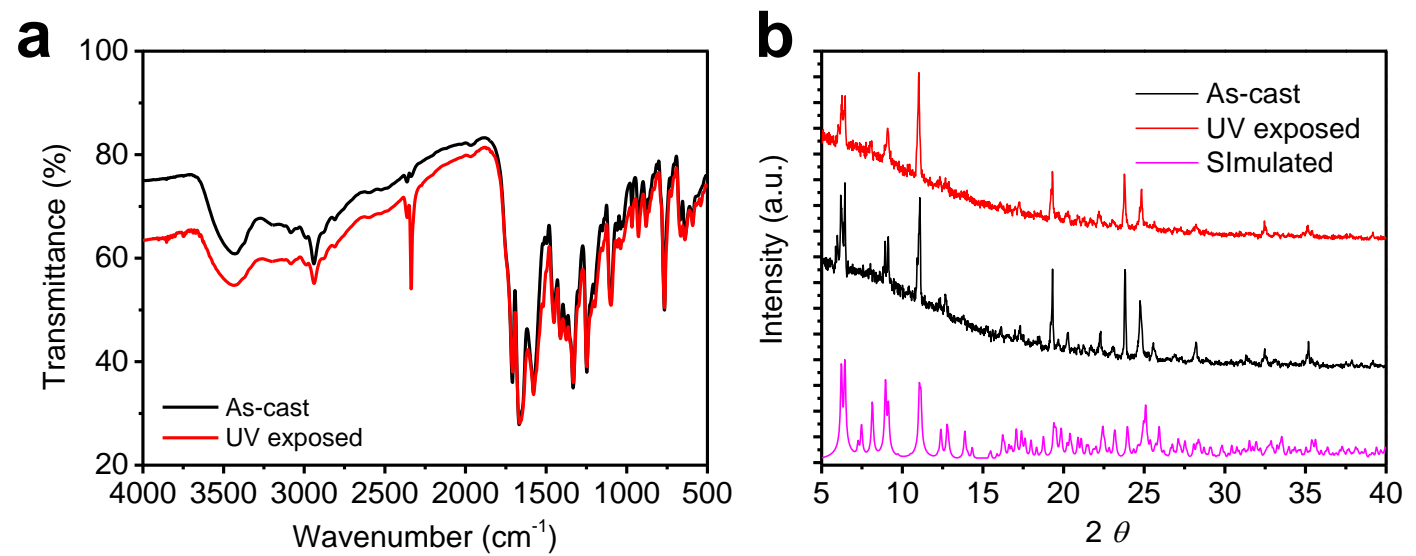

Figure S5. (a) FT-IR results of (Rac)-AlaNDI-Cd SCNs before and after the exposure UV-light for $1 \mathrm{~h}$ under UV light was exposed $(\lambda=365 \mathrm{~nm}, 150 \mu \mathrm{W} \mathrm{cm}-2)$. (b) PXRD results of heterochiral AlaNDI-Cd SCNs before and after UV light was exposed for $1 \mathrm{~h}(\lambda=365 \mathrm{~nm}, 150$ $\mu \mathrm{W} \mathrm{cm}{ }^{-2}$ ) with simulated PXRD results from the crystal structure. 


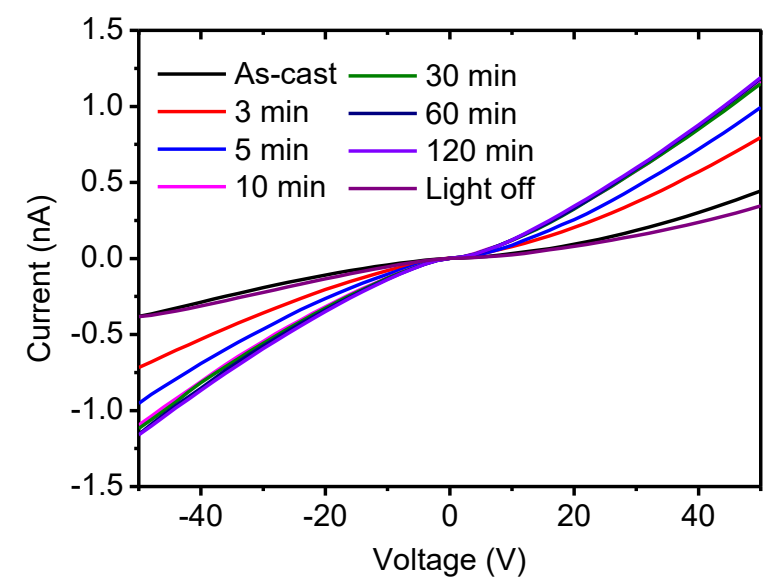

Figure S6. $I-V$ curves of (Rac)-AlaNDI-Cd micro/nanocrystals under different UV light exposure time of up to $2 \mathrm{~h}$ and in dark condition. 

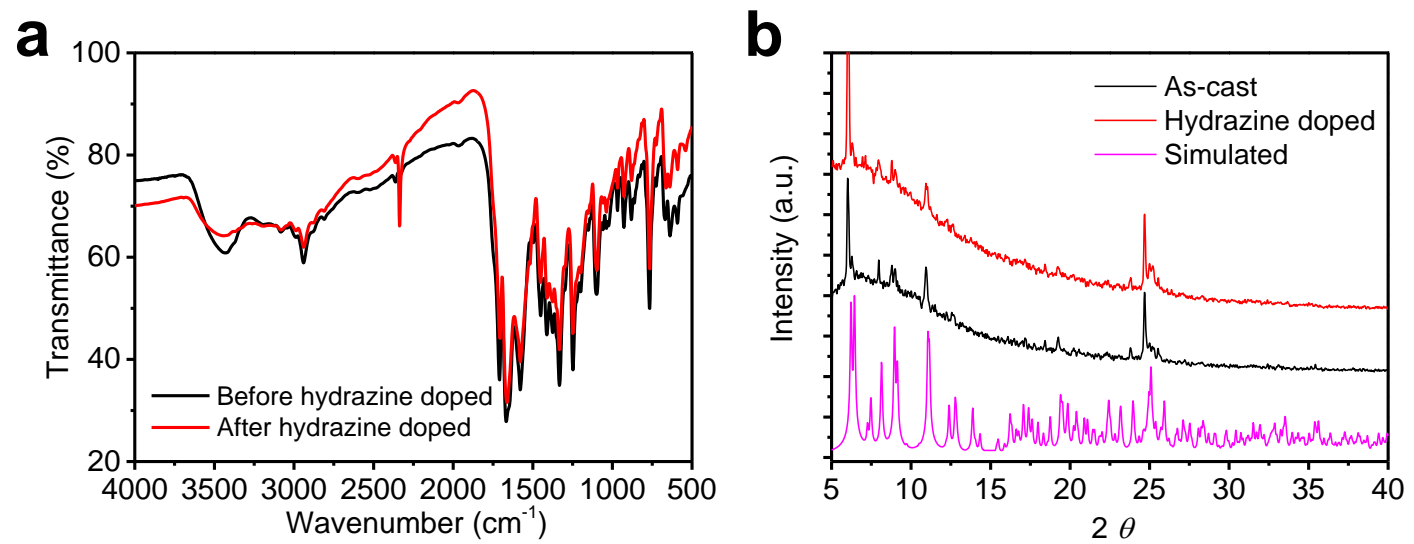

Figure S7. (a) FT-IR results of heterochiral AlaNDI-Cd SCNs before and after hydrazine doping for 5 min. (b) PXRD results of heterochiral AlaNDI-Cd SCNs before and after hydrazine doping for $5 \mathrm{~min}$ with simulated PXRD results from the crystal structure. 


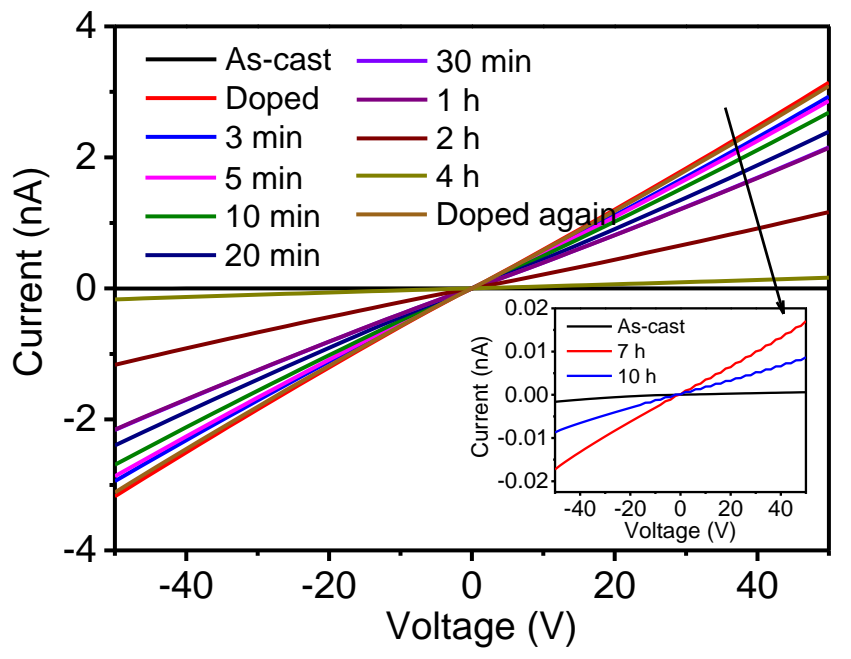

Figure S8. $I-V$ characteristics of hydrazine doped heterochiral AlaNDI-Cd micro/nanocrystals depending on the passed time after hydrazine doping in ambient condition. 
a

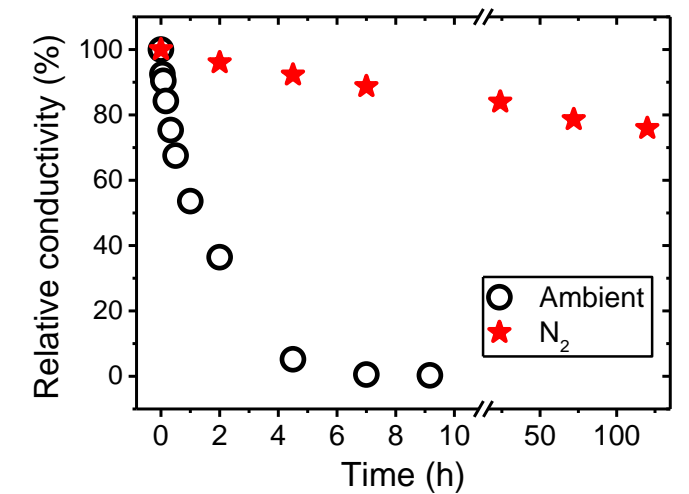

C

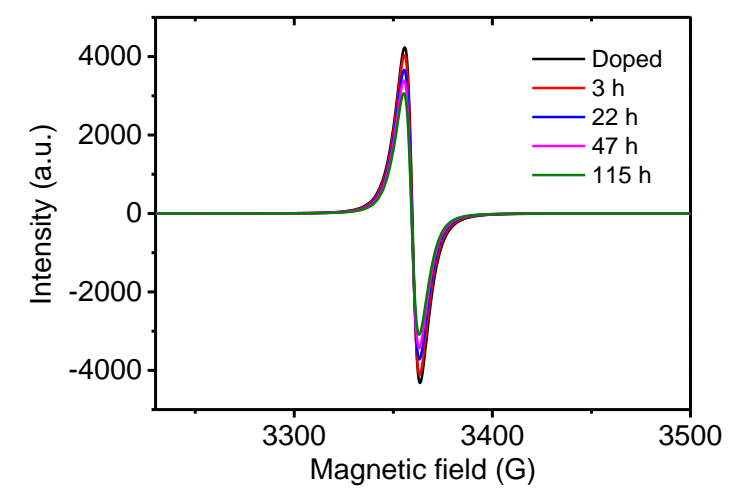

b

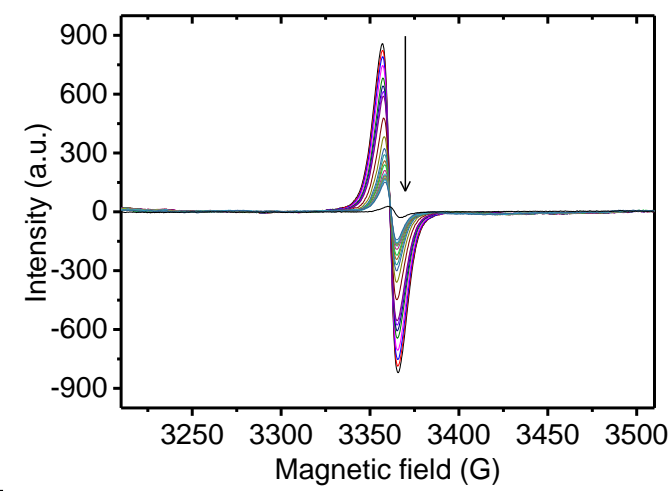

d

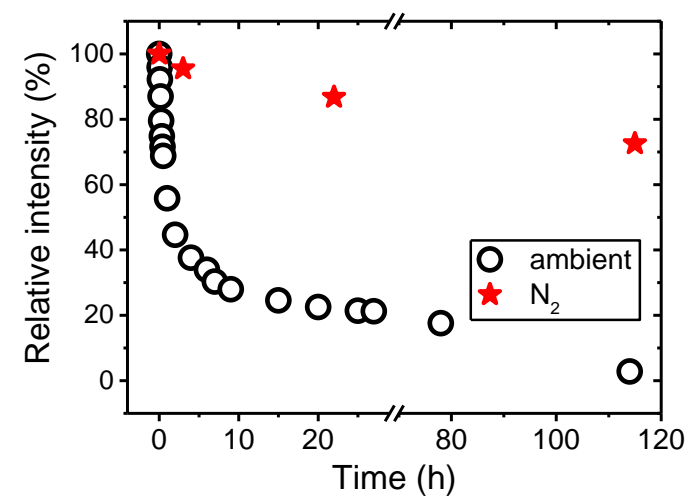

Figure S9. (a) Conductivity changes of hydrazine doped heterochiral AlaNDI-Cd micro/nanocrystals in ambient condition and $\mathrm{N}_{2}$ atmosphere. (b) ESR spectra of hydrazine doped heterochiral AlaNDI-Cd micro/nanocrystals as time passed; 3 min, 5 min, 10 min, 15 min, 20 min, 25 min, 30 min, 1 h, 2 h, 4 h, 6 h, 7 h, 9 h, 15 h, 20 h, 25 h, 27 h, 37 h, 45 h, 50 h, 60 h, 78 h, 114 h. (c) ESR spectra of hydrazine doped heterochiral AlaNDI-Cd micro/nanocrystals in $\mathrm{N}_{2}$ glove box as time passed. (d) Time-dependent ESR intensity of hydrazine doped heterochiral AlaNDI-Cd micro/nanocrystals at $3359 \mathrm{G}$ in ambient condition and $\mathrm{N}_{2}$ atmosphere. 


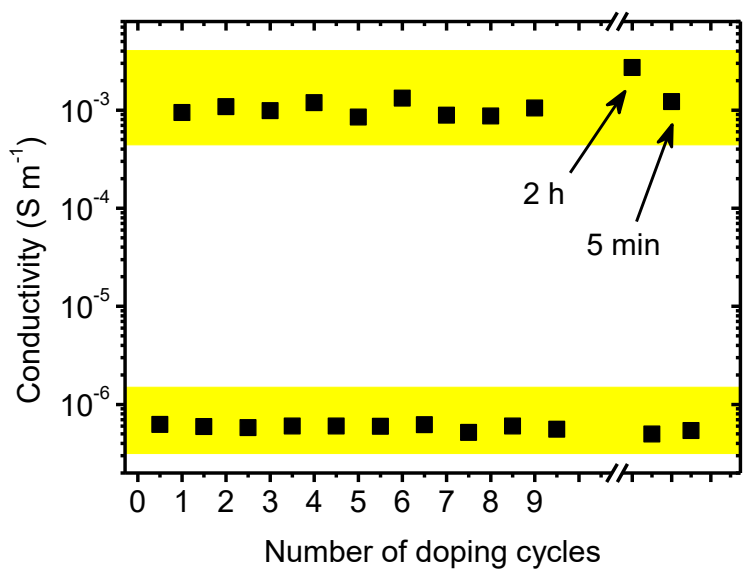

Figure S10. Cyclic stability of conductivity reversibility of (Rac)-AlaNDI-Cd micro/nanocrystals. The initial 10 cycles were conducted with repeated hydrazine doping for 5 min and exposure to high heat in vacuum condition $\left(100^{\circ} \mathrm{C}, 1 \mathrm{~h}\right)$ under ambient condition. 


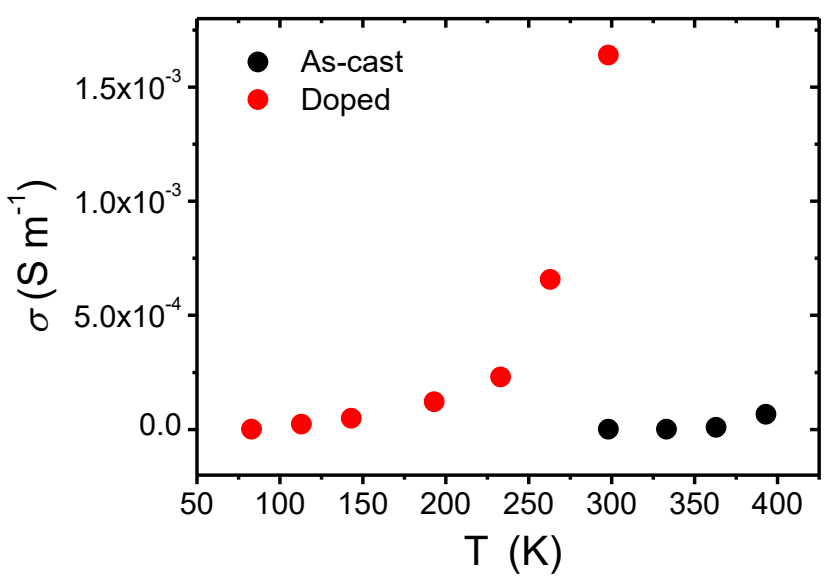

Figure S11. Temperature-dependent conductivity of heterochiral AlaNDI-Cd micro/nanocrystals before and after hydrazine doping. 


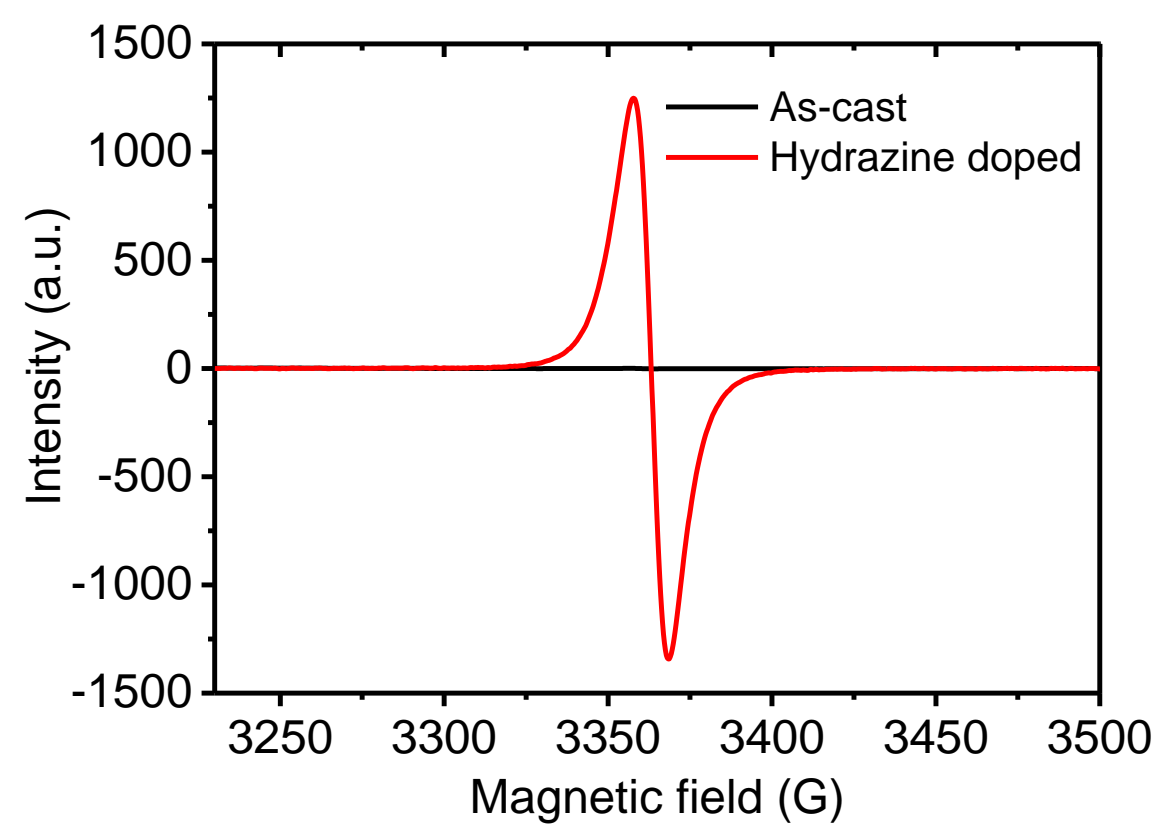

Figure S12. ESR spectra of homochiral $(S)$-AlaNDI-Cd micro/nanocrystals before and after hydrazine doping. 


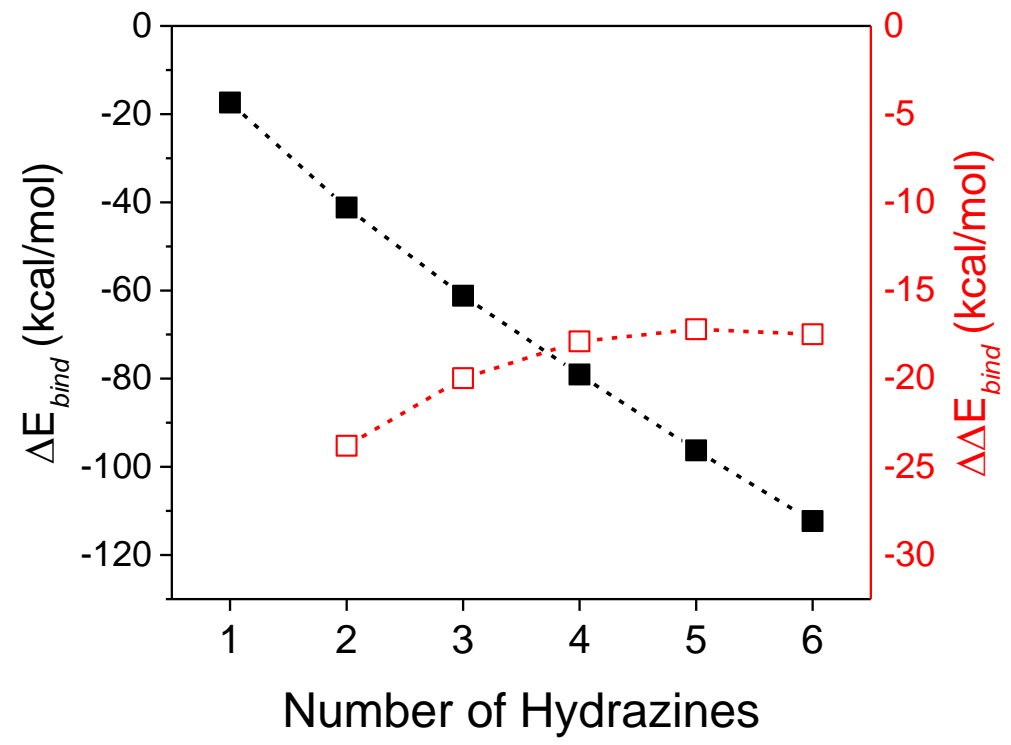

Figure S13. Binding energy calculation for adsorbed hydrazine. The binding energy $\left(\Delta E_{b i n d}\right.$, black line $)$ and differential binding energy $\left(\Delta \Delta E_{\text {bind, }}\right.$, red line $)$ with increasing number of adsorbed hydrazine molecules on the (Rac)-AlaNDI-Cd surface. 

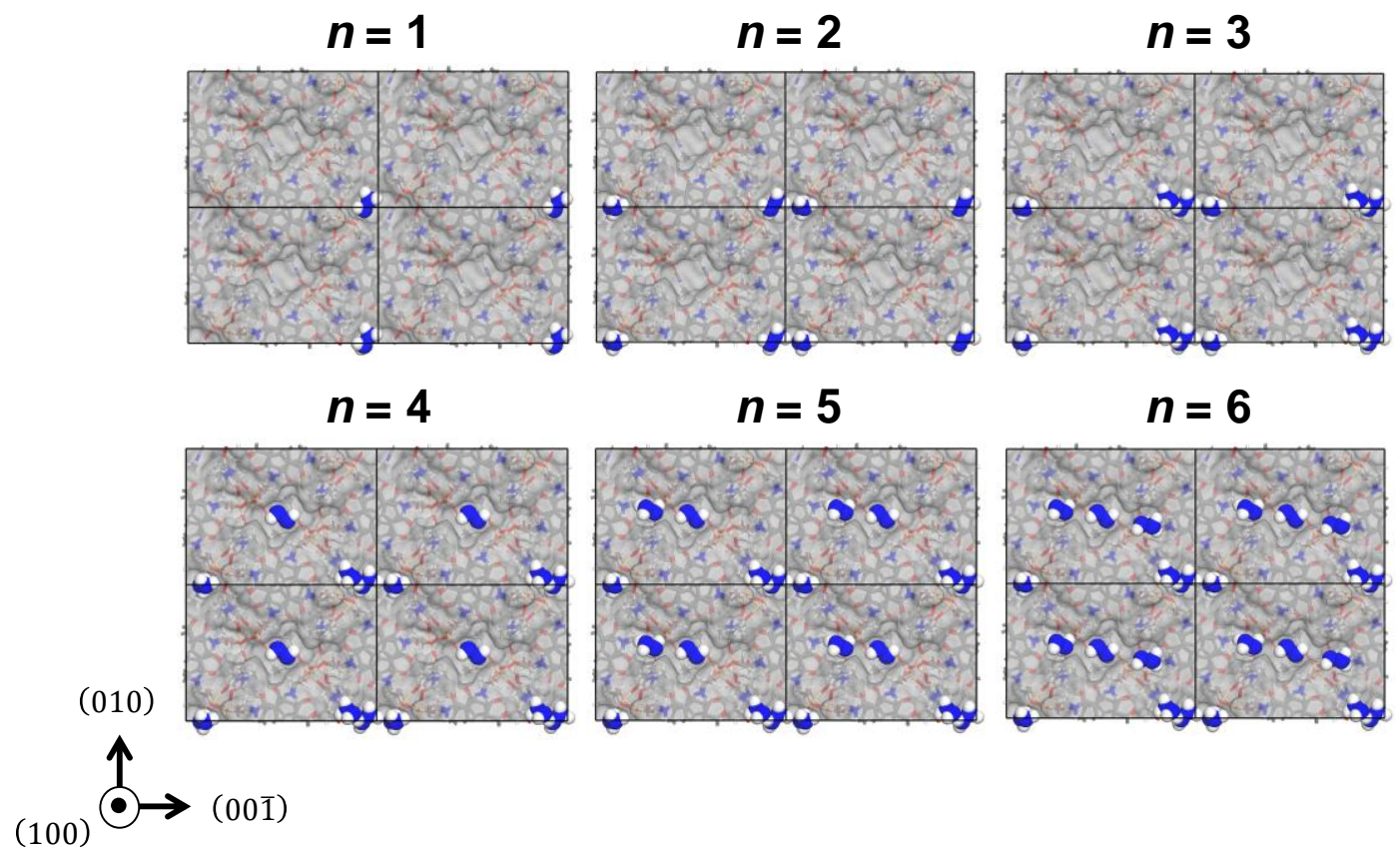

Figure S14. Binding configurations of AlaNDI-Cd with adsorbed hydrazine. Binding configurations of $(R a c)$-AlaNDI-Cd surface with increasing number of adsorbed hydrazine molecules. For the clear view, surface system was represented as $2 \times 2 \times 1$ supercell and the Connolly surface of (Rac)-AlaNDI-Cd was also presented (gray colored region). The carbon, hydrogen, oxygen, nitrogen, and cadmium atoms are colored in gray, white, red, blue, and ivory, respectively. 